\title{
Energy-Efficient Improper Signaling for $K$-User Interference Channels
}

\author{
Mohammad Soleymani*, Christian Lameiro*, Ignacio Santamaria ${ }^{\dagger}$ and Peter J. Schreier* \\ *Signal \& System Theory Group, Universität Paderborn, Germany \\ ${ }^{\dagger}$ Dept. Communications Engineering, Universidad de Cantabria, Spain \\ Email: \{mohammad.soleymani, christian.lameiro, peter.schreier\}@sst.upb.de, i.santamaria@unican.es
}

\begin{abstract}
This paper investigates the energy efficiency (EE) of improper Gaussian signaling (IGS) in a $K$-user interference channel (IC). IGS allows unequal variances and/or correlation between the real and imaginary parts, and it has recently been shown to be advantageous in various interference-limited scenarios. In this paper, we propose an energy-efficient IGS design for the $K$-user IC, which is based on a separate optimization of the powers and complementary variances of the users. We compare the EE region achieved by the proposed scheme with that achieved by conventional proper signaling and show that IGS can significantly improve the EE region.

Index Terms-Energy efficiency, improper Gaussian signaling, interference channels, multiuser.
\end{abstract}

\section{INTRODUCTION}

Energy-aware techniques have become more and more important in the design of modern wireless communication systems. For instance, energy efficiency is among the main concerns of future $5 \mathrm{G}$ networks [1]. Another primary issue in modern wireless communication systems, which are fundamentally interference-limited, is how to handle interference. Therefore, interference management techniques play an essential role in the design of such systems.

Proper Gaussian signaling (PGS) achieves the capacity of point-to-point, broadcast, and multiple-access channels [2]. However, this is not the case in other interference-limited systems, such as the $K$-user interference channel (IC). It is in these scenarios where improper Gaussian signaling (IGS) has been shown to bring performance improvements over PGS. IGS has been used as an effective interference management tool in various interference-limited systems [3]-[10]. In IGS schemes, the real and imaginary parts of the signals are correlated and/or have unequal power [11].

The benefits of IGS as an interference management tool have been shown using different performance metrics. For instance, IGS schemes may increase the achievable degreesof-freedom (DoF) of the system, which are a proxy for the sum-rate capacity when the signal-to-noise ratio (SNR) is large [3]. Also, IGS schemes have been shown to improve the achievable rate for various interference-limited systems when the interference is treated as noise at the recievers [4]-[10]. In [4], [5], it was shown that IGS can increase the achievable rate of a single-input single-output (SISO) 2-user IC. The paper [8] showed that IGS can enlarge the achievable rate region of a multiple-input single-output (MISO) $K$-user IC.
The energy efficiency (EE) of a user is defined as the ratio of its achievable rate to its total power consumption [12][15]. There are many works on EE in PGS (see, e.g., [1], [12] and the references therein). In [12], energy-efficient PGS schemes were considered for different scenarios such as pointto-point communications, the $K$-user SISO IC, relay multipleinput multiple-output systems, etc. Nevertheless, the number of works considering EE for IGS systems is small. In [10], we proposed an energy-efficient IGS for 2-user underlay cognitive radio (UCR) systems and showed the benefits of IGS for the secondary user from an EE perspective. In UCR, there are two types of users with different priority, i.e., primary and secondary users, which do not cooperate with each other. This is in contrast with the $K$-user IC, in which the users have the same priority and can cooperate to achieve a better performance.

In this paper, we tackle the optimization of EE of IGS schemes in the $K$-user IC, and study the potential benefits of IGS from this point of view. We aim at deriving the EE region of IGS for the $K$-user IC by casting the corresponding optimization problem as maximizing the weighted minimum EE (WMEE) [12]. To solve this optimization problem, we employ a sequential optimization method, in which we first maximize the WMEE over the powers considering PGS, i.e., when the complementary variances are zero. We then maximize the WMEE over the complementary variances for the given powers. This suboptimal approach guarantees that the proposed IGS scheme is not worse than the PGS scheme. Our results show that IGS can substantially enlarge the EE region of the $K$-user IC.

The rest of this paper is organized as follows. Section II describes the system model and formulates the WMEE problem. Sections III and IV present the energy-efficient schemes for PGS and IGS, respectively. Section V presents some numerical results.

\section{SYSTEM MODEL}

\section{A. Preliminaries of IGS}

Let us consider a zero-mean complex Gaussian random variable $x$ with variance $p=\mathbb{E}\left\{|x|^{2}\right\}$ and complementary variance $q=\mathbb{E}\left\{x^{2}\right\}$. We define the circularity coefficient of $x$ as $\kappa=\frac{|q|}{p}$, where $0 \leq \kappa \leq 1$ or equivalently $|q| \leq p$ [11]. We call $x$ proper if $\kappa=0$, and improper otherwise. We denote the probability distribution of such $x$ by $\mathcal{C N}(0, p, q)$. 


\section{B. Problem Statement}

We consider a $K$-user IC, as depicted in Fig. 1 . The received signal of user $k \in\{1, \cdots, K\}$ is

$$
y_{k}=\sum_{j} h_{k j} x_{j}+n_{k},
$$

where $x_{j}, h_{k j}$, and $n_{k}$ for $j \in\{1, \cdots, K\}$ are the transmit signal of user $j$, channel coefficient of the link between transmitter $j$ and receiver $k$, and independent zero-mean proper complex Gaussian noise with variance $\sigma^{2}$, respectively. We treat interference as noise and assume that each user is allowed to employ IGS. Thus, the rate of user $k$ is [5]

$$
R_{k}=\frac{1}{2} \log _{2}\left(\frac{\left(\sigma^{2}+\sum_{j} p_{j}\left|h_{k j}\right|^{2}\right)^{2}-\left|\sum_{j} q_{j} h_{k j}^{2}\right|^{2}}{\left(\sigma^{2}+\sum_{j \neq k}\left|h_{k j}\right|^{2} p_{j}\right)^{2}-\left|\sum_{j \neq k} q_{j} h_{k j}^{2}\right|^{2}}\right),
$$

where $p_{j}$, and $q_{j}$ are, respectively, the transmission power, and complementary variance of the transmitted signal of user $j$. We can represent the rate of user $k$ in vector form as

$$
R_{k}(\mathbf{p}, \mathbf{q})=\frac{1}{2} \log _{2}\left(\frac{\left(\sigma^{2}+\mathbf{a}_{k}^{T} \mathbf{p}\right)^{2}-\left|\mathbf{f}_{k}^{H} \mathbf{q}\right|^{2}}{\left(\sigma^{2}+\mathbf{b}_{k}^{T} \mathbf{p}\right)^{2}-\left|\mathbf{g}_{k}^{H} \mathbf{q}\right|^{2}}\right)
$$

where

$$
\begin{aligned}
& \mathbf{a}_{k}=\left[\begin{array}{llll}
\left|h_{k 1}\right|^{2} & \left|h_{k 1}\right|^{2} & \cdots & \left|h_{k K}\right|^{2}
\end{array}\right]^{T}, \\
& \mathbf{b}_{k}=\mathbf{a}_{k}-\left[\begin{array}{llllll}
0 & 0 & \cdots & \left|h_{k k}\right|^{2} & \cdots & 0
\end{array}\right]^{T} \text {, } \\
& \mathbf{f}_{k}=\left[\begin{array}{llll}
h_{k 1}^{2} & h_{k 2}^{2} & \cdots & h_{k K}^{2}
\end{array}\right]^{H}, \\
& \mathbf{g}_{k}=\mathbf{f}_{k}-\left[\begin{array}{llllll}
0 & 0 & \cdots & h_{k k}^{2} & \cdots & 0
\end{array}\right]^{H}, \\
& \mathbf{q}=\left[\begin{array}{llll}
q_{1} & q_{2} & \cdots & q_{K}
\end{array}\right]^{H}, \\
& \mathbf{p}=\left[\begin{array}{llll}
p_{1} & p_{2} & \cdots & p_{K}
\end{array}\right]^{T} .
\end{aligned}
$$

We define the energy efficiency function of user $k$ as the ratio of its data rate to its total transmission power, i.e.,

$$
U_{k}=\frac{R_{k}(\mathbf{p}, \mathbf{q})}{\zeta p_{k}+P_{c}},
$$

where $\zeta$ and $P_{c}$ are the power efficiency and the constant power consumed by each transmitter, respectively [12]-[15].

In this paper, we aim at deriving the EE region of the $K$-user IC, which can be cast as maximizing the weighted minimum EE (WMEE) as [12]

$$
\begin{gathered}
\max _{\mathbf{p}, \mathbf{q}} \min _{k}\left\{\frac{U_{k}}{\alpha_{k}}=\frac{R_{k}(\mathbf{p}, \mathbf{q})}{\alpha_{k}\left(\zeta p_{k}+P_{c}\right)}\right\}, \\
\text { s.t. } \quad 0 \leq p_{k} \leq P_{k}, \\
\left|q_{k}\right| \leq p_{k},
\end{gathered}
$$

where $\left(\alpha_{k}\right)^{-1}$ is the corresponding weight for user $k$, and $\sum_{k=1}^{K} \alpha_{k}=1$. Moreover, $P_{k}$ is the power budget of user $k$. Solving (11) is not straightforward since it is not a convex optimization problem. In order to solve (11), we employ a sequential optimization approach, in which we first obtain the powers that maximize the WMEE problem for $\mathbf{q}=\mathbf{0}$. To this

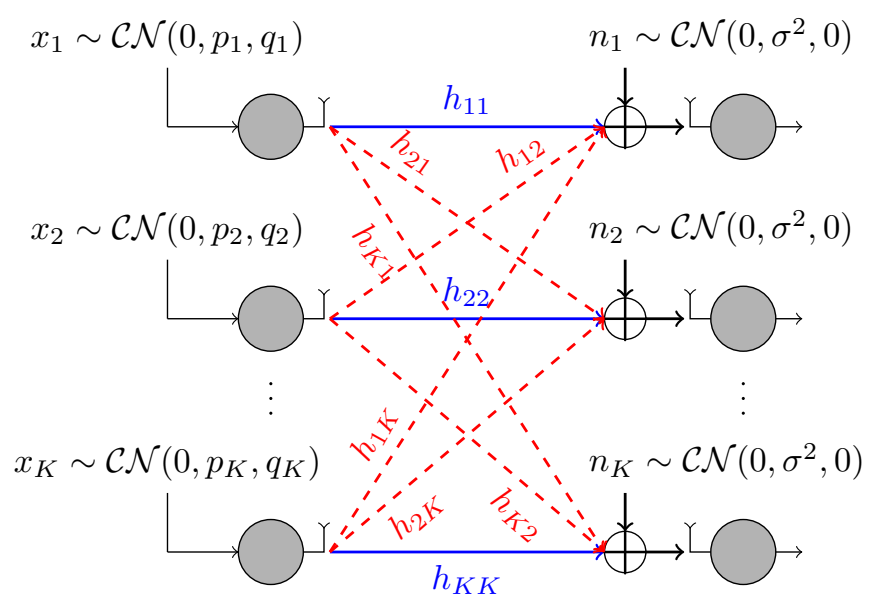

Fig. 1: Channel model for the $K$-user SISO IC.

end, we employ the PGS scheme in [12]. Then, we derive the complementary variances by optimizing (11) for the given power vector.

\section{OPTIMIZATION OF THE TRANSMIT POWERS}

In this section, we obtain the powers, $\mathbf{p}$, that maximize the WMEE cost function when users employ PGS, i.e., $\mathbf{q}=\mathbf{0}$. In this case, (11) can be simplified to

$$
\begin{gathered}
\max _{\mathbf{p}} \min _{k}\left\{\frac{U_{k}^{p}(\mathbf{p})}{\alpha_{k}}=\frac{R_{k}^{p}(\mathbf{p})}{\alpha_{k}\left(\zeta p_{k}+P_{c}\right)}\right\}, \\
\text { s.t. } \quad 0 \leq p_{k} \leq P_{k},
\end{gathered}
$$

where $U_{k}^{p}(\mathbf{p})$ and $R_{k}^{p}(\mathbf{p})=\log _{2}\left(1+\gamma_{k}(\mathbf{p})\right)$ are, respectively, the EE and rate of user $k$ when all users employ PGS. Moreover, $\gamma_{k}(\mathbf{p})$ is the received signal-to-interference-plusnoise ratio (SINR) at the receiver of user $k$, which is given by

$$
\gamma_{k}(\mathbf{p})=\frac{p_{k}\left|h_{k k}\right|^{2}}{\sigma^{2}+\sum_{j \neq k}\left|h_{k j}\right|^{2} p_{j}} .
$$

The optimization problem (12) is not convex, but a suboptimal solution was proposed in [12]. For the sake of completeness, we describe the solution here. We first apply the generalized Dinkelbach algorithm (GDA) to (12). Then, we solve the optimization problem in each iteration of the GDA by a sequence of convex problems. Due to space restrictions, we refer the reader to [12], [16] for more details about the GDA.

Applying the GDA to (12) results in the following optimization problem at the $l$ th iteration

$$
\begin{aligned}
\max _{\mathbf{p}} \min _{k} & \log _{2}\left(1+\gamma_{k}(\mathbf{p})\right)-\mu^{(l)} \alpha_{k}\left(\zeta p_{k}+P_{c}\right) \\
\text { s.t. } & 0 \leq p_{k} \leq P_{k},
\end{aligned}
$$

where $\mu^{(l)}=U_{k}^{p}\left(\mathbf{p}^{(l-1)}\right)$ is fixed at each iteration, and $\mathbf{p}^{(l-1)}$ is the power vector at the $(l-1)$ th iteration. Unfortunately, (14) is not convex and needs further simplification. To this end, we first approximate the objective function of (14) by a lower bound by using the following inequality [12]

$$
\log _{2}\left(1+\gamma_{k}(\mathbf{p})\right) \geq a^{(l)} \log _{2}\left(\gamma_{k}(\mathbf{p})\right)+b^{(l)},
$$


where

$$
\begin{aligned}
a^{(l)}= & \frac{\gamma_{k}\left(\mathbf{p}^{(l-1)}\right)}{1+\gamma_{k}\left(\mathbf{p}^{(l-1)}\right)}, \\
b^{(l)}= & \log _{2}\left(1+\gamma_{k}\left(\mathbf{p}^{(l-1)}\right)\right) \\
& \quad-\frac{\gamma_{k}\left(\mathbf{p}^{(l-1)}\right) \log _{2}\left(\gamma_{k}\left(\mathbf{p}^{(l-1)}\right)\right)}{1+\gamma_{k}\left(\mathbf{p}^{(l-1)}\right)} .
\end{aligned}
$$

Note that (15) holds with equality at $\gamma_{k}(\mathbf{p})=\gamma_{k}\left(\mathbf{p}^{(l-1)}\right)$. Moreover, the derivatives of the left-hand side and right-hand side of (15) are equal at $\gamma_{k}\left(\mathbf{p}^{(l-1)}\right)$. As a result, we can replace the logarithmic part of the objective function in (14) with its lower bound and apply sequential convex programming (SCP) to solve (14). Note that under these conditions, SCP converges to a point satisfying the Karush-Kuhn-Tucker (KKT) conditions of (14) [17]. Hence, we approximate (14) as [12]

$$
\begin{array}{ll}
\max _{\mathbf{p}, e} & e \\
\text { s.t. } & a_{k}^{(l)} \log _{2}\left(p_{k}\left|h_{k k}\right|^{2}\right)-a_{k}^{(l)} \log _{2}\left(\sigma^{2}+\sum_{j \neq k}\left|h_{k j}\right|^{2} p_{j}\right) \\
& +b_{k}^{(l)}-\mu^{(l)} \alpha_{k}\left(\zeta p_{k}+P_{c}\right) \geq e, \quad \forall k, \\
& 0 \leq p_{k} \leq P_{k},
\end{array}
$$

Now we substitute $p_{k}$ by $t_{k}=\log _{2}\left(p_{k}\right)$ and rewrite (17) as [12]

$$
\begin{array}{ll}
\max _{\mathbf{t}, e} & e \\
\text { s.t. } & a_{k}^{(l)} t_{k}+\log _{2}\left(\left|h_{k k}\right|^{2}\right)-a_{k}^{(l)} \log _{2}\left(\sigma^{2}+\sum_{j \neq k}\left|h_{k j}\right|^{2} 2^{t_{j}}\right) \\
& +b_{k}^{(l)}-\mu^{(l)} \alpha_{k}\left(\zeta 2^{t_{k}}+P_{c}\right) \geq e, \quad \forall k, \\
& 0 \leq t_{k} \leq \log _{2}\left(P_{k}\right),
\end{array}
$$

where $\mathbf{t}=\left[t_{1}, t_{2}, \ldots, t_{k}\right]$. The problem (18) is convex in $\left\{t_{k}\right\}_{k=1}^{K}$ and can be solved efficiently.

\section{OPTIMIZING COMPLEMENTARY VARIANCES}

In this section, we maximize the WMEE function over $\mathbf{q}$ for the given $\mathbf{p}^{\star}$ obtained in Section III. The corresponding optimization problem is again nonconvex and difficult to solve. Thus, we employ an iterative algorithm to find a suboptimal solution. This is accomplished by approximating the original optimization problem and solving each approximated problem by a sequence of convex feasibility problems.

For a given power vector $\mathbf{p}^{\star}$, (11) is equivalent to

$$
\begin{aligned}
& \max _{\mathbf{q}, e} e \\
& \text { s.t. } \quad R_{k}\left(\mathbf{p}^{\star}, \mathbf{q}\right) \geq \alpha_{k}\left(\zeta p_{k}^{\star}+P_{c}\right) e, \quad \forall k, \\
& \left|q_{k}\right| \leq p_{k}^{\star} \text {, } \\
& \forall k \text {. }
\end{aligned}
$$

We can rewrite (19) as

$$
\begin{array}{rlr}
\max _{\mathbf{q}, e} & e \\
\text { s.t. } & -\left(\left(\sigma^{2}+\mathbf{b}_{k}^{T} \mathbf{p}^{\star}\right)^{2}-\left|\mathbf{g}_{k}^{H} \mathbf{q}\right|^{2}\right) 2^{2 \alpha_{k}\left(\zeta p_{k}^{\star}+P_{c}\right) e} \\
& +\left(\sigma^{2}+\mathbf{a}_{k}^{T} \mathbf{p}^{\star}\right)^{2}-\left|\mathbf{f}_{k}^{H} \mathbf{q}\right|^{2} \geq 0, \quad \forall k, \\
& \left|q_{k}\right| \leq p_{k}^{\star}, & \forall k .
\end{array}
$$

The optimization problem in (20) is not convex since (20b) is not a concave function in $\mathbf{q}$ and $e$. Following along lines similar to problem (14), we first find a lower bound for (20b), such that it is a concave function in $\mathbf{q}$ for a fixed $e$. That is, we approximate the nonconvex part of (20b) by an affine function of $\mathbf{q}$, using the first-order Taylor expansion at $\mathbf{q}^{(m)}$, i.e., [11]

$$
\left|\mathbf{g}_{k}^{H} \mathbf{q}\right|^{2} \geq\left|\mathbf{g}_{k}^{H} \mathbf{q}^{(m)}\right|^{2}+2 \Re\left(\left(\mathbf{g}_{k}^{H} \mathbf{q}^{(m)}\right)^{H} \mathbf{g}_{k}^{H}\left(\mathbf{q}-\mathbf{q}^{(m)}\right)\right),
$$

where $\mathbf{q}^{(m)}$ is the solution obtained at the previous iteration, and $\mathfrak{R}(x)$ takes the real part of $x$. Note that the equality holds only at $\mathbf{q}=\mathbf{q}^{(m)}$. Furthermore, the derivatives of the left-hand side and right-hand side of (21) are equal at $\mathbf{q}^{(m)}$. Under these conditions, the approximated problem converges to a point satisfying the KKT conditions of (20) [17]. By plugging (21) into (20), the approximated optimization problem at the $m$ th iteration becomes

$$
\begin{array}{cll}
\max _{\mathbf{q}, e} & e \\
\text { s.t. } & \tilde{E}^{(m)}(\mathbf{q}, e)>0, \quad \forall k, \\
& \left|q_{k}\right| \leq p_{k}^{\star}, \quad \forall k,
\end{array}
$$

where

$$
\begin{aligned}
\tilde{E}^{(m)}(\mathbf{q}, e) & =2^{1+2 \alpha_{k}\left(\zeta p_{k}^{\star}+P_{c}\right) e} \mathfrak{R}\left(\left(\mathbf{g}_{k}^{H} \mathbf{q}^{(m)}\right)^{H} \mathbf{g}_{k}^{H}\left(\mathbf{q}-\mathbf{q}^{(m)}\right)\right) \\
+ & 2^{2 \alpha_{k}\left(\zeta p_{k}^{\star}+P_{c}\right) e}\left|\mathbf{g}_{k}^{H} \mathbf{q}^{(m)}\right|^{2}+\left(\sigma^{2}+\mathbf{a}_{k}^{T} \mathbf{p}^{\star}\right)^{2} \\
& -\left|\mathbf{f}_{k}^{H} \mathbf{q}\right|^{2}-2^{2 \alpha_{k}\left(\zeta p_{k}^{\star}+P_{c}\right) e}\left(\sigma^{2}+\mathbf{b}_{k}^{T} \mathbf{p}^{\star}\right)^{2} .
\end{aligned}
$$

The optimization problem (22) is not convex, but we can obtain its optimal solution, $e^{(m)}$, by a bisection method, which results in a sequence of feasibility problems [18]. That is, we fix $e=e_{0}$ and solve

$$
\begin{array}{rll}
\text { find } & \mathbf{q} \\
\text { s.t. } & \tilde{E}^{(m)}\left(\mathbf{q}, e_{0}\right)>0, \quad \forall k, \\
& \left|q_{k}\right| \leq p_{k}^{\star}, & \forall k .
\end{array}
$$

If (24) is feasible, $e^{(m)}>e_{0}$. Otherwise, $e^{(m)}<e_{0}$. As indicated formerly, the complete algorithm converges to a point satisfying the KKT conditions of (20).

\section{NUMERICAL RESULTS}

In this section, we provide some numerical examples to illustrate the EE improvement achieved by IGS. We set $\sigma^{2}=$ $10^{-4} \mathrm{~W}, \zeta=3$, and $P_{k}=5 \mathrm{~mW}$ for $k=1, \ldots, K$ [15]. We average the results over 100 channel realizations. Every channel realization is drawn from a proper complex Gaussian distribution with zero mean and unit variance, i.e., $\mathcal{C N}(0,1,0)$. 


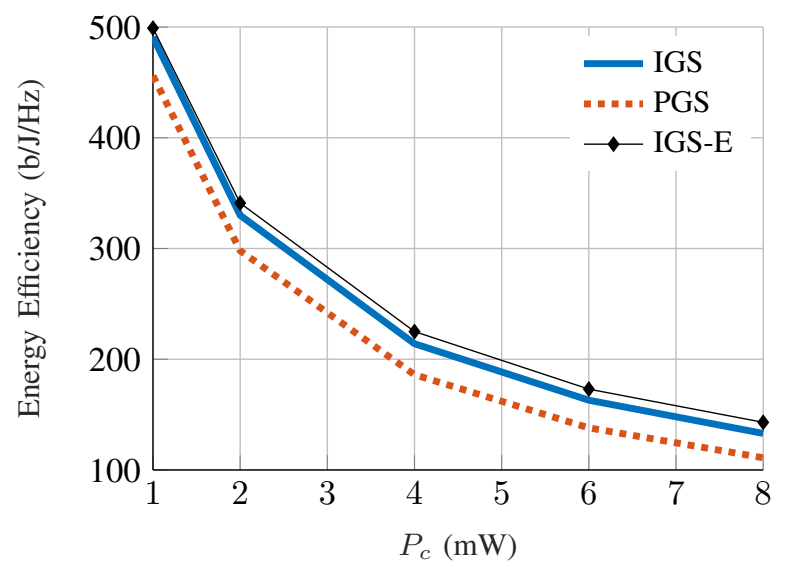

Fig. 2: Symmetric EE of the 2-user IC versus $P_{c}$.

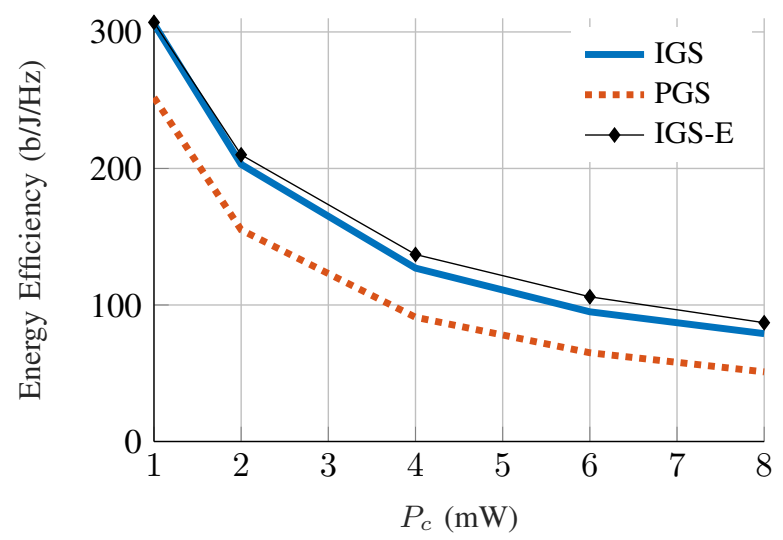

Fig. 3: Symmetric EE of the 3-user IC versus $P_{c}$.

In Figs. 2 and 3, we show the fairness point of the EE region boundary, which is given by $\alpha_{k}=1 / K$, versus $P_{c}$ for the 2user and 3-user IC, respectively. Notice that at this point the users achieve the same EE, and therefore it is typically denoted as symmetric EE. These figures show that IGS provides higher average symmetric EE than PGS. As expected, the EE decreases with $P_{c}$. In these figures, we also compare our proposed IGS scheme with an IGS scheme based on exhaustive search with $5 \times 10^{5}$ and $10^{7}$ randomizations (labeled by IGSE) for the 2-user and 3-user IC, respectively. This provides a hint on how close the proposed IGS suboptimal design is to the optimal solution. As can be observed, the proposed IGS scheme performs very close to IGS-E in this scenario. In particular, for the example in Fig. 2, our IGS scheme performs only $1.5 \%$ and $7.5 \%$ worse than the IGS-E at $P_{c}=1 \mathrm{~mW}$ and $P_{c}=8 \mathrm{~mW}$, respectively. Similarly, for the example in Fig. 3 , the IGS-E is $0.3 \%$ and $11.2 \%$ better than our scheme when $P_{c}=1 \mathrm{~mW}$ and $P_{c}=8 \mathrm{~mW}$, respectively. Note that the IGS-E can be considered a lower bound for the optimal IGS solution.

In Fig. 4, we show the improvement in the average symmetric EE by IGS versus $P_{c}$ for the 2-user and 3-user ICs. As can be observed, the improvement by IGS increases with $P_{c}$. The reason is that the optimal powers for PGS increase with $P_{c}$,

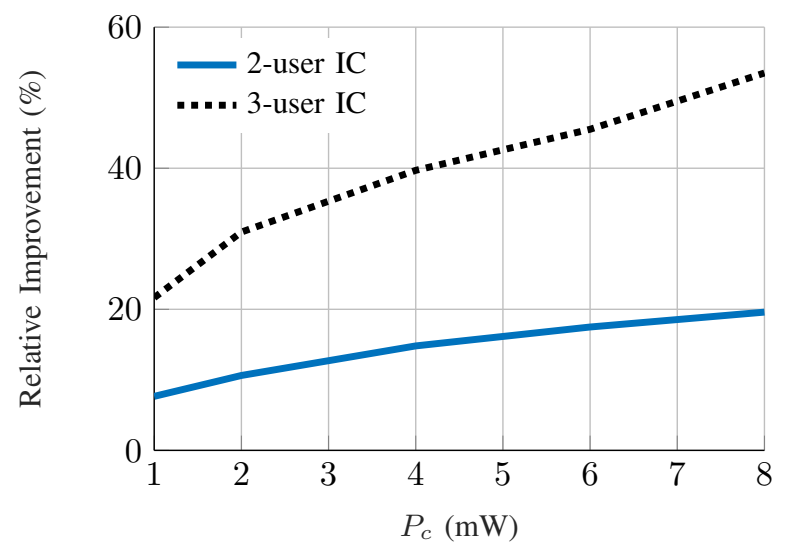

Fig. 4: Relative improvement by employing IGS for the symmetric EE versus $P_{c}$.

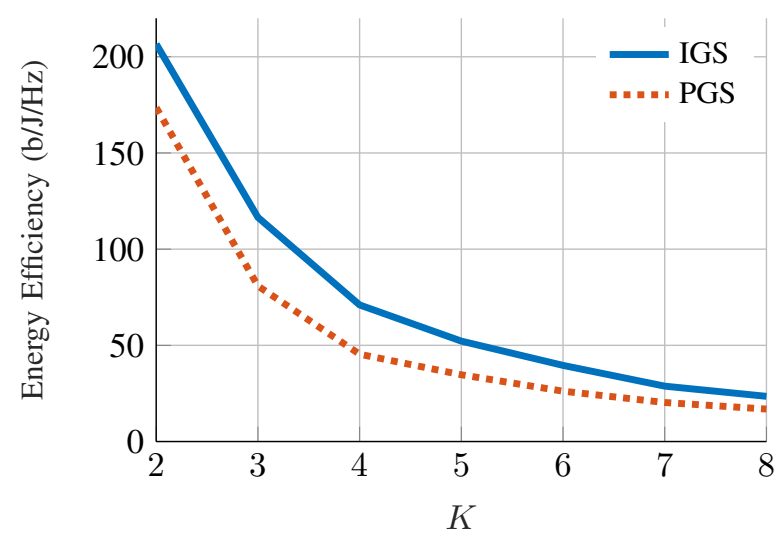

Fig. 5: Symmetric EE of the $K$-user IC versus $K$.

and the EE maximization is simplified to rate maximization when $P_{c}$ is very high [1]. Since higher powers result in more improvement by optimizing over complementary variances [5], the improvement is also increasing with $P_{c}$. We can also observe in Fig. 4 that the improvement by IGS is more significant for the 3-user IC. This is due to the fact that the higher the number of users is, the more interference they cause. As a result, the benefits of IGS are potentially higher in the 3-user IC.

In Fig. 5, we show the symmetric EE versus the number of users, $K$, for $P_{c}=4 \mathrm{~mW}$. As can be seen, the symmetric EE decreases with $K$. In Fig. 6, we show the sum of the symmetric EE versus the number of users, $K$, for $P_{c}=4 \mathrm{~mW}$. As expected, the sum of the symmetric EE decreases with $K$, which implies that the overall EE decreases when more users employ the same amount of resources. In these figures, we can observe that IGS significantly outperforms PGS for every $K$, where there is $19 \%$ and $56 \%$ improvement for $K=2$ and $K=4$, respectively.

Figure 7 shows the EE region of the 2-user IC for $P_{c}=5$ $\mathrm{mW}$ and channel realization

$$
\mathbf{H}=\left[\begin{array}{cc}
1.3997 e^{-i 0.3803} & 1.5401 e^{i 1.0127} \\
1.2250 e^{i 2.4311} & 0.9361 e^{i 0.8844}
\end{array}\right] .
$$

For this channel realization, the interference link of each 


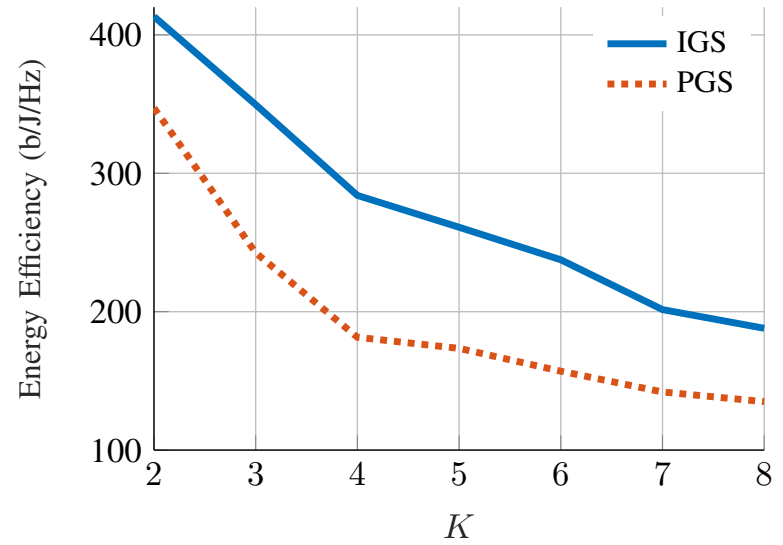

Fig. 6: Sum of symmetric EE of the $K$-user IC versus $K$.

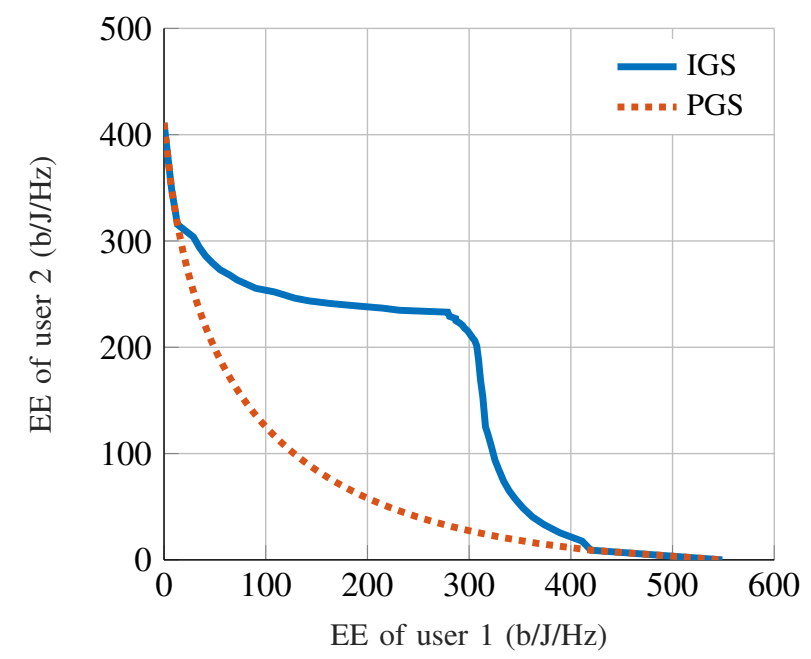

Fig. 7: Energy efficiency region of of the 2-user IC for channel realization $\mathbf{H}$.

receiver is stronger than its corresponding direct link, which yields a strong interference regime. As can be observed, IGS can significantly enlarge the EE region for this example. It is worth mentioning that IGS does not provide any gain in noise-limited regimes, for which the interference is negligible. In other words, not surprisingly the benefits of IGS increase with the interference level.

\section{CONCLUSION}

We have proposed an EE IGS scheme for the $K$-user IC. By maximizing the WMEE, the proposed design permits operating at different points of the EE region. In order to find a suboptimal solution to the WMEE problem, we have performed a separate optimization of the powers and complementary variances. For the former, we have considered proper signaling and resorted to GDA and SCP to find a solution to the original non-convex problem satisfying the KKT conditions. The resulting solution was then improved by optimizing over the complementary variances. Again, this has resulted in a non-convex problem, and a suboptimal solution was found by using bisection and SCP. Our numerical results show that IGS is much more energy efficient than its proper counterpart.

\section{ACKNOWLEDGMENT}

The work of M. Soleymani, C. Lameiro and P. J. Schreier was supported by the German Research Foundation (DFG) under grants SCHR1384/8-1 and LA 4107/1-1. The work of I. Santamaria was supported by the Ministerio de Economia y Competitividad (MINECO) and AEI/FEDER funds of the UE, Spain, under project CARMEN (TEC2016-75067-C4-4-R).

\section{REFERENCES}

[1] S. Buzzi, I. Chih-Lin, T. E. Klein, H. V. Poor, C. Yang, and A. Zappone, "A survey of energy-efficient techniques for $5 \mathrm{G}$ networks and challenges ahead," IEEE J. Sel. Areas Commun., vol. 34, no. 4, pp. 697-709, 2016.

[2] T. M. Cover and J. A. Thomas, Elements of Information Theory. John Wiley \& Sons, 2012.

[3] V. R. Cadambe, S. A. Jafar, and C. Wang, "Interference alignment with asymmetric complex signaling-Settling the Høst-Madsen-Nosratinia conjecture," IEEE Trans. Inf. Theory, vol. 56, no. 9, pp. 4552-4565, 2010.

[4] Z. K. Ho and E. Jorswieck, "Improper Gaussian signaling on the two-user SISO interference channel," IEEE Trans. Wireless Commun., vol. 11, no. 9, pp. 3194-3203, 2012.

[5] Y. Zeng, C. M. Yetis, E. Gunawan, Y. L. Guan, and R. Zhang, "Transmit optimization with improper Gaussian signaling for interference channels," IEEE Trans. Signal Process., vol. 61, no. 11, pp. 2899-2913, 2013.

[6] C. Lameiro, I. Santamaría, and P. J. Schreier, "Benefits of improper signaling for underlay cognitive radio," IEEE Wireless Commun. Lett., vol. 4, no. 1, pp. 22-25, 2015.

[7] O. Amin, W. Abediseid, and M.-S. Alouini, "Underlay cognitive radio systems with improper Gaussian signaling: Outage performance analysis," IEEE Trans. Wireless Commun., vol. 15, no. 7, pp. 4875-4887, 2016.

[8] Y. Zeng, R. Zhang, E. Gunawan, and Y. L. Guan, "Optimized transmission with improper Gaussian signaling in the $K$-user MISO interference channel," IEEE Trans. Wireless Commun., vol. 12, no. 12, pp. 63036313, 2013.

[9] M. Soleymani, C. Lameiro, I. Santamaría, and P. J. Schreier, "Robust improper signaling for two-user SISO interference channels," IEEE Trans. Commun., 2019.

[10] M. Soleymani, C. Lameiro, P. J. Schreier, and I. Santamaría, "Energyefficient design for underlay cognitive radio using improper signaling," in Proc. IEEE Int. Conf. on Acoust., Speech and Signal Processing (ICASSP), 2019, pp. 4769-4773.

[11] P. J. Schreier and L. L. Scharf, Statistical Signal Processing of ComplexValued Data: the Theory of Improper and Noncircular Signals. Cambridge University Press, 2010.

[12] A. Zappone and E. Jorswieck, "Energy efficiency in wireless networks via fractional programming theory," Found Trends in Commun. Inf. Theory, vol. 11, no. 3-4, pp. 185-396, 2015.

[13] C. Isheden, Z. Chong, E. Jorswieck, and G. Fettweis, "Framework for link-level energy efficiency optimization with informed transmitter," IEEE Trans. Wireless Commun., vol. 11, no. 8, pp. 2946-2957, 2012.

[14] Y. Chen, S. Zhang, and S. Xu, "Impact of non-ideal efficiency on bits per Joule performance of base station transmissions," in Proc. IEEE VTC - Spring, 2011, pp. 1-5.

[15] J. Mao, G. Xie, J. Gao, and Y. Liu, "Energy efficiency optimization for OFDM-based cognitive radio systems: A water-filling factor aided search method," IEEE Trans. Wireless Commun., vol. 12, no. 5, pp. 2366-2375, 2013.

[16] W. Dinkelbach, "On nonlinear fractional programming," Management Science, vol. 13, no. 7, pp. 492-498, 1967.

[17] A. Aubry, A. De Maio, A. Zappone, M. Razaviyayn, and Z.-Q. Luo, "A new sequential optimization procedure and its applications to resource allocation for wireless systems," IEEE Trans. Signal Process., vol. 66, no. 24, pp. 6518-6533.

[18] S. Boyd and L. Vandenberghe, Convex Optimization. Cambridge University Press, 2004. 